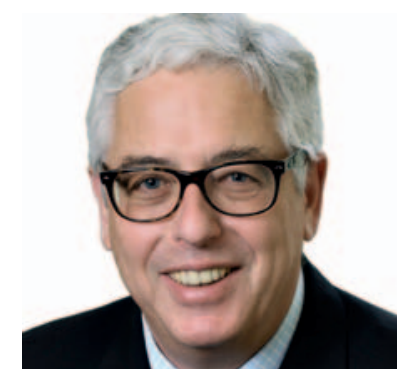

\title{
Aktinische Keratose: Photodynamische Therapie mit MAL-PDT zeigt Überlegenheit gegenüber Ingenolmebutat
}

\section{Percy Lehmann}

HELIOS Universitätsklinikum Wuppertal, Dermatologie - Hautklinik

Abstractübersetzung aus Zane C, Fabiano A, Arisi M, Calzavara-Pinton P: A randomized split-face clinical trial of photodynamic therapy with methyl aminolevulinate versus ingenol mebutate gel for the treatment of multiple actinic keratoses of the face and scalp. Dermatology 2016;232:472-477.

Randomisierte klinische Studie mit Halbseitenvergleich zur photodynamischen Therapie mit

Methylaminolävulinat versus Ingenolmebutat-Gel in der Behandlung multipler aktinischer Keratosen des Gesichts und der Kopfhaut

\section{Schlüsselwörter}

Aktinische Keratose · Photodynamische Therapie .

Methylaminolävulinat · Ingenolmebutat-Gel

\section{Zusammenfassung}

Hintergrund: Photodynamische Therapie in Verbindung mit Methylamino-Lävulinsäure (MAL-PDT) und mit IngenolmebutatGel (IMB) sind zugelassene therapeutische Optionen bei multiplen aktinischen Keratosen (AK).

Ziel: Das Ziel dieser randomisierten klinischen Studie mit intraindividuellem Halbseitenvergleich war es, die Behandlungsergebnisse von MAL-PDT und IMB zu vergleichen.
Methoden: Zwei symmetrische kontralaterale Areale mit einer vergleichbaren Zahl AK-Läsionen wurden ausgewählt und per Randomisierung der Behandlung entweder mit einem 3-tägigen IMB-Zyklus oder einer einzelnen MAL-PDT-Sitzung zugeteilt. Am nächsten Tag wurde ein Score zur Beurteilung der lokalen Hautreaktionen (LHR) erhoben. Die Patienten beurteilten die Schmerzen und die Heilungsdauer der behandelten Areale.

Ergebnisse: Nach 90 Tagen wurden der Anteil der Läsionen in Vollremission, die Anzahl der Patienten mit Vollremission aller Läsionen, das kosmetische Ergebnis und die Präferenz des Patienten beurteilt.

Schlussfolgerung: Unseren Ergebnissen zufolge zeigten IMB und MAL-PDT vergleichbare Wirksamkeit, allerdings war das kosmetische Ergebnis nach MAL-PDT besser. Die Schmerzen waren unter PDT stärker, aber unter IMB waren die LHR ausgeprägter und die Heilung dauerte länger. Die Patienten bevorzugten die MAL-PDT.

(c) 2017 S. Karger GmbH, Freiburg

\section{KARGER}

Fax +4976145207 14 information@karger.com www.karger.com 


\section{Transfer in die Praxis}

\section{Hintergrund}

Die photodynamische Therapie mit Methylamino-Lävulinsäure (MAL-PDT) hat seit 2003 einen festen Platz in der Therapie von aktinischen Keratosen. Neben chirurgischen bzw. physikalischen Methoden und vom Patienten selbst anzuwendenden Topica ist die Durchführung der MAL-PDT inzwischen eine etablierte effektive Methode zur Behandlung des nicht-melanozytären Hautkrebses. Unter den Topica hat insbesondere Ingenolmebutat (Picato ${ }^{\circledR}$ ) große Beachtung gefunden, zumal die topische Applikation nur an 3 Tagen erfolgt und konsekutiv die beschriebenen Ergebnisse als gut bis sehr gut eingestuft werden können. Fehlend ist bislang eine direkte Vergleichsstudie (Head-to-Head-Studie), wie sie in der vorliegenden Arbeit beschrieben wird.

\section{Studienergebnisse}

Es handelt sich um eine randomisierte Halbseitenstudie, die die photodynamische Therapie mit Methylamino-Lävulinsäure $\left(\right.$ Metvix ${ }^{\circledR}$ ) mit Ingenolmebutat (Picato ${ }^{\circledR}$ ) vergleicht. Diese Studie ist wertvoll, weil die Entscheidung, welche der zahlreichen zur Verfügung stehenden Therapieoptionen beim einzelnen Patienten mit Feldkanzerisierung angebracht ist, keine einfache ist. Die Studie wurde sauber konzipiert. Es erfolgt im Halbseitenvergleich die Anwendung der zwei oben beschriebenen Standardprozeduren an 35 Patienten. Es zeigte sich, dass die Prozedur der photodynamischen Therapie mit Metvix ${ }^{\circledR}$ akut schmerzhafter war als die Applikation der topischen Substanz Picato ${ }^{\circledR}$, auf längere Sicht aber besser vertragen wurde.

Im Behandlungsergebnis war die MAL-PDT effektiver als die Behandlung mit Ingenolmebutat, wobei eine statistische Signifikanz hier nicht erreicht wurde. Das kosmetische Ergebnis war jedoch signifikant besser mit der photodynamischen Therapie. Hier spielt wahrscheinlich eine Rolle, dass die photodynamische Therapie auch gegenüber sonstigen Zeichen der chronischen Lichtschädigung wirksam ist.

Bezüglich der Nebenwirkungen war die MAL-PDT anfangs schmerzhafter, jedoch war die längerfristige Hautentzündung durch Ingenolmebutat schwerwiegender, und die Abheilungsphase dauerte länger. In der Folge bevorzugten die Patienten in den meisten Fällen die photodynamische Therapie gegenüber der topischen Therapie. Aufgrund der Einschränkungen der Fläche wurde in dieser Studie nur eine Region von $25 \mathrm{~cm}^{2}$ behandelt wie es für die Anwendung von Picato ${ }^{\circledR}$ vorgesehen ist. Allerdings können weitaus größere Flächen mittels PDT behandelt werden.

\section{Fazit für die Praxis}

Insgesamt erscheint also aufgrund der vorliegenden Daten dieser Studie die konventionelle photodynamische Therapie gegenüber der Applikation von Ingenolmebutat überlegen, insbesondere, wenn man die Patientenpräferenz, das kosmetische Ergebnis und die Flächeneinschränkungen berücksichtigt.

\section{Disclosure Statement}

Hiermit erkläre ich, dass ich im Advisory Board von Galderma beratend tätig bin.

Kontaktadresse: Prof. Dr. med. Percy Lehmann, HELIOS Universitätsklinikum Wuppertal, Dermatologie - Hautklinik, Heusnerstraße 40, Haus 5 und 1, 42283 Wuppertal, Deutschland, percy.lehmann@helios-kliniken.de 